\title{
Thermal and Multifunctional Properties of Nanoparticles
}

\author{
Mashal Alenazi \\ University of Bridgeport, Biomedical Engineering, USA
}

\begin{abstract}
Monodisperse nickel oxide (NiO) nanoparticles with tunable size have been synthesized by hot-injection method. The NiO nanoparticles were characterized by $\mathrm{X}$-ray diffraction, transmission electron micro-scopy, ultraviolet and visible spectrophotometer. There are numerous techniques for microfabrication of patterned polymer surfaces and microchips for drug delivery. While silicon has been the choice material for much of the research done with MEMS, the methacrylates and acrylates provide a rapid and inexpensive base for future work. Cyclodextrins have been used for two purposes: as a solubilising agent of paclitaxel, which it is a very lipophilic compound, and for their ability to disturb and inhibit the activity of the intestinal P-gp.
\end{abstract}

Keywords: Thermal, hydrothermal, multifunctional, Nanoparticles.

\section{INTRODUCTION}

Recently, nanoparticles have become attractive objects for life science applications, in particular, in such rapidly growing areas as express diagnostics and advanced medical treatment. Encapsulation of nanoparticles with drug molecules $(1,2)$ or attaching them to viruses, bacteria, etc. are of special interest. Time-controlled release of the absorbed drugs would be advantageous for treatment of many diseases, e.g. diabetes, because of a decreased number of injections compared to that of molecular insulin. Furthermore, fluorescent or colored particles such as quantum dots (QD) (3), nanodiamonds (4), and gold nanoparticles (5) can be used for diagnostics as markers that provide visual information about the distribution of labeled agents in tissues and blood. Magnetic particles (MP) (6) can be also used as efficient labels for MRI diagnostics and can be precisely quantified even inside a living organism by an external induction probe $(7,8)$. At present, MP are widely studied for hyperthermia of tumors by heating in an $\mathrm{AC}$ magnetic field and for targeted delivery of drugs by magnetic field gradients, to avoid systemic intoxication of the organism $(9,10)$. Specific immunological targeting of nanoparticles by antibodies against pathogenic cells is another noteworthy application. Not only does it allow marking tumors for accurate dissection, but it also enhances drug delivery to the target cells.

Anosized materials have attracted worldwide attentions owing to the excellent functional properties and promising technology application [1]. The nickel oxide $(\mathrm{NiO})$ is obviously p-type semiconductor and has a relatively wide band gap energy in the range of 3.6-4.0 eV among the various oxides materials [2]. Because of its unique electronic structure [3], NiO nanoparticles have an extensively application research field [4], such as photocatalysts [5], photovoltaic devices [6], gas sensors [7], solar cell [8] and electrochromic film [9]. A lot of synthetic method has been introduced to synthesize $\mathrm{NiO}$ nanoparticles [10], such as sol-gel method [11], spray pyrolysis [12], sputtering [13], microemulsion method [14] and hydrothermal technique. Different from the above synthesis techniques, the hot-injection method has some advantages such as simple process, less hazardous, relatively mild condition, low cost [16], and easily to produce high purity products. Moreover, it is excellent that the synthesized nanoparticles have good uniformity and monodispersity. Timonen et al. synthesized cobalt nanoparticles by injecting dicobalt octacarbonyl. Zhang et al. [18] found that CdS NCs were controllably synthesized by adjusting reaction conditions through a hot-injection method. He application of nanoparticles in numerous areas is attracting more and more attention due to unusual physical and chemical properties exhibited by them such as high surface areato-volume ratio and catalytic potential which makes them better in comparison to their bulk materials $[1,2]$. Among various nano-particles, magnetic nanoparticles especially nanosized ferrite materials have been frequently used in variety of fields such as biosensors, gas sensors, magnetic catalysis, transformer core drug delivery, magnetic resonance imaging (MRI), lithium ion batteries, magnetic materials and microwave devices [3-5]. Cobalt ferrite $(\mathrm{CoFe} 2 \mathrm{O})$ is one of the most widely used ferrites because of its high coercivity, chemical stability, mechanical hardness, moderate saturation magnetization and photo-induced magnetic effects [1,6-9]. Though variety of applications of cobalt ferrite have been investigated, but its application as an adsorbent material for the treatment of metallic pollutants is not yet much explored. Some studies regarding the application of nanosized cobalt ferrite in the treatment of metals and dyes from simulated samples are available, but those regarding its application for industrial effluents are very rare.

Controllable fabrication of inorganic-organic polymer hybrid composites based on the molecular level has been 
extensively investigated due to their potential applications, drugs that are marketed is limited at this point. Recently, such as electrochemical sensors, drug carriers and much attention has been focused on nanoemulsions and nanocomposites materials. However, it seems to be self-emulsified drug delivery systems (SEDDS) to difficult to obtain well-dispersed inorganic-organic improve the oral bioavailability of poorly water-soluble nanocomposites owing to the strong tendency of drugs. Nanoemulsions are non-equilibrium, heterogeneous aggregation among inorganic nanoparticles, which may systems consisting of two immiscible liquids in which one serve to depress properties of nanocomposites. To this liquid is dispersed as droplets within the other liquid [58]. end, considerable efforts have been devoted to the design Self-nanoemulsified drug delivery systems are isotropic and controlled fabrication of well-defined inorganicorganic polymer hybrids.

\section{CORE DRUG ADHESION}

To confirm microstructure and purity of the prepared final products, XRD patterns of as-prepared samples with different amount of oleic acid and different reaction temperatures illustrates the products with various amount of oleic acid. It can be seen that the diffraction peaks of the nanoparticles can be well index to the standard $\mathrm{NiO}$ spectrum (JCPDS card, No. 47-1049) by using $1 \mathrm{ml}$ oleic acid. When the amount of oleic acid were $2 \mathrm{ml}$ and $3 \mathrm{ml}$, the diffraction peaks of the nanoparticles can be well index to the Ni (JCPDS card, No. 040850). Therefore, the optimum oleic acid amount for the preparation of $\mathrm{NiO}$ nanoparticles was $1 \mathrm{ml}$. Keeping the amount of oleic acid $1 \mathrm{ml}$, we changed the reaction temperature in the hotinjection. Fig. 1(b) shows the XRD patterns of as-prepared nanoparticles grown at $240{ }^{\circ} \mathrm{C}, 260{ }^{\circ} \mathrm{C}$ and $280{ }^{\circ} \mathrm{C}$.

Bragg peaks at $37.25^{\circ}, 43.28^{\circ}, 62.88^{\circ}, 75.41^{\circ}$ and $79.41^{\circ}$ were indexed to the (111), (200), (220), (311) and (222) planes, respectively.

All the diffraction peaks of nanoparticles are well agreement with the standard $\mathrm{NiO}$ spectrum and there are no any other diffraction peaks of impurities were observed, indicating that the final nanoparticles is highly purity $\mathrm{NiO}$ phase. The main sizes of nanoparticles were calculated by using Debye-Scherrer formula in the range 5-100 nm. The pure diffraction peak widths at half height became narrow with the increase of temperature, indicating that the size of $\mathrm{NiO}$ nanoparticles became larger as the reaction temperature increase. Therefore, the reaction temperature plays a critical role in controlling the particle size of the $\mathrm{NiO}$ nanoparticles.

The notion that the era of nanomedicines started in the previous century is demonstrated when looking at the development of nanocrystal technology, discussed by Eugene Cooper. Rather simple wet milling techniques led to a decrease of the crystal size down to the hundred nanometer range. These dispersions are stabilized with surface active polymers. The oral bioavailability of nanocrystal based formulations of poorly water-soluble drugs has been clearly enhanced for some drugs. Poorly water-soluble nanocrystals were also used for parenteral injection of imaging material for CT scans. The 'nanocrystal story' clearly shows that rather simple techniques can improve the performance of poorly watersoluble drugs dramatically, although the number of such mixtures of oil, surfactant, co-surfactant and drug that form fine oil-in-water $(\mathrm{o} / \mathrm{w})$ nanoemulsions when introduced into aqueous phases under gentle agitation. They are stabilized by an interfacial film of surfactant molecules with a droplet size typically less than $100 \mathrm{~nm}$, which guarantees efficient absorption of oil droplets.

Nanostructural magnetic materials have the potential to revolutionize current data storage technologies, magnetoelectronics, and biotechnology. Recently, the surface and size effects in magnetic nanoparticles have been one of the main topics among scientists. The core/shell systems,in which both of the core and shell are magnetic, are gaining increasing attention due to their appealing novel properties and promising application. Especially, the research about ferromagnetic (FM)/antiferromagnetic (AFM) core/shell structures has become a focus. Many valuable phenomena, such as giant magnetoresistance and interfacial exchange bias effect, have been found in the structures. Basically, nanosized transition group metal particles are usually chosen to be the magnetic cores. Compared with Feand Co, the synthesized Ni particles are especially commended due to the antioxidant ability. So far, several physical and chemical composite methods, such as sonochemical deposition, colloidal chemistry method [15], mechanical milling, polyol method and chemical reduction have been applied to fabricate the nanosized $\mathrm{Ni}$ particles. As exemplified in the polyol method which is a typical chemically composite route, $\mathrm{Ni}(\mathrm{NO})$ was dissolved in an organic solvent like polyethylene glycol (PEG) and then heated to a certain temperature. An organic surfactant like oleic acid or oleyl amine was used to prevent the $\mathrm{Ni}$ nanoparticles from agglomeration. The changes of melting enthalpy, H, of the PLA/MMT system prepared by the melt extrusion were considerably higher than those obtained by solvent dissolution method. It was considered that the binding force between PLA and inorganic compound in the composite prepared by melt extrusion method was higher than in those prepared by solvent dissolution method. The automotive industry makes frequent use of the cast multicomponent hypoeutectic $\mathrm{AlSiCu}$ alloys. These alloys have been characterized by presence of two $\mathrm{Al}-\mathrm{Si}$ and $\mathrm{Al}-\mathrm{Si}-\mathrm{Cu}$ eutectics, which are primarily responsible for defining the microstructure and mechanical properties of these alloys. Comprehensive understanding of solidification paths of these alloys is of paramount importance for metallurgical engineers. This knowledge also enable the process, quality and simulation engineers as well as designers to ensure that the casting will achieve the desired properties for its intended application after corresponding melting, liquid metal 
processing, mold filling and heat treatment procedure. In order to ensure that cast components have good mechanical properties their as-cast microstructures must be closely monitored.

\section{CONCLUSION}

He saturation magneti- zation of nanoparticles at room temperature was determined to be $50.8 \mathrm{Am} 2 \mathrm{~kg} 1$.Magnetic remanence and saturation magnetization were recorded to be $18.98 \mathrm{Am} 2 \mathrm{~kg} 1$ and $50.8(\mathrm{Am} 2 / \mathrm{kg})$ respectively.It was observed that removal $(\%)$ decreased on increasing the initial concentration of adsorbate. However, $\mathrm{CoFe} 2 \mathrm{O}$ nanoparticles showed very promising results even for higher $\mathrm{Cr}(\mathrm{VI})$ concentration ranges $(150 \mathrm{mgL} 14)$. In the case of printing press wastewater, $\mathrm{Cr}(\mathrm{VI})$ removal was $67 \%$ and it was also efficient for the removal of other available metallic species. Increased removal was observed at a higher temperature for synthetic wastewater as well as printing press wastewater, which suggested the endothermic adsorption of $\mathrm{Cr}(\mathrm{VI})$ ions. The molecular basis of the GI drug barrier has been found mainly due to the overexpression of the multidrug efflux pump proteins, P-glycoproteins (P-gp) in the epithelial cell membrane. Additionally, carriers in the particulate form should be able to diffuse further into the mucus layer enabling them to reach the cells of the epithelial layer. The particle size and surface properties, namely, their relative hydrophobicity, are the main factors affecting the particles'

\section{REFERENCES}

[1] Mei, L., Zhang, Z., Zhao, L., Huang, L., Yang, X.-L., Tang, J., \& Feng, S.-S. (2013). Pharmaceutical nanotechnology for oral delivery of anticancer drugs. Advanced Drug Delivery Reviews, 65(6), 880-890.

[2] Peppas, N. A. (2004). Intelligent therapeutics: biomimetic systems and nanotechnology in drug delivery. Advanced Drug Delivery Reviews, 56(11), 1529-1531.

[3] Wingender, J., Neu, T. R., \& Flemming, H. C. (Eds.). (2012). Microbial extracellular polymeric substances: characterization, structure and function. Springer Science \& Business Media.

[4] Hajji, P., David, L., Gerard, J. F., Pascault, J. P., \& Vigier, G. (1999). Synthesis, structure, and morphology of polymer-silica hybrid nanocomposites based on hydroxyethyl methacrylate. Journal of Polymer Science Part B: Polymer Physics, 37(22), 31723187.

[5] [3] Burnside, S. D., \& Giannelis, E. P. (1995). Synthesis and properties of new poly (dimethylsiloxane) nanocomposites. Chemistry of materials, 7(9), 1597-1600

[6] S. Cao, W. Zeng, H. Long, J. Gong, Z. Zhu, L. Chen, Synthesis and controlled

[7] growth of $\mathrm{NiO}$ hierarchical bundle-like nanoflowers with the assistance of

[8] Ethylene glycol, Mater. Lett. 161 (2015) 275-277.

[9] Y. Lu, Y.H. Ma, S.Y. Ma, W.X. Jin, S.H. Yan, X.L. Xu, et al., Synthesis of cactus-like

[10] $\mathrm{NiO}$ nanostructure and their gas-sensing properties, Mater. Lett. 164 (2016)

[11] 48-51.

[12] P. Subalakshmi, A. Sivashanmugam, Fuel aided synthesis of NiO flakes for

[13] Electrochemical energy storage application, J. Alloy. Compd. 662 (2016)200-207. 